\title{
Liquid Propellant Mass Measurement in Microgravity
}

Kevin M. Crosby ${ }^{1}$, Rudy J. Werlink², Eric A. Hurlbert ${ }^{3}$

${ }^{1}$ Department of Physics and Astronomy, Carthage College, 2001 Alford Park Drive, Kenosha, WI 53140; ${ }^{2}$ ASA Kennedy Space Center; ${ }^{3}$ NASA Johnson Space Center

Abstract

The Modal Propellant Gauging (MPG) experiment has demonstrated sub-1\% gauging accuracy under laboratory conditions on both flight hardware and subscale tanks. Recently, MPG was adapted for flight on Blue Origin's New Shepard vehicle and has flown twice, achieving equilibrated, zero-g surface configurations of propellant simulant at three different fill fractions. Flight data from MPG missions on New Shepard P7 and P9 show agreement between known and measured propellant levels of $0.3 \%$ for the fill fractions investigated in the present study. Two approaches for estimating zero-g propellant mass are described here. Both approaches rely on measuring shifts in modal frequencies of a tank excited by acoustic surface waves and subject to fluid mass loading by the propellant. In the first approach, shifts in the lowest mode frequency (LMF) are measured and associated with liquid fill-level changes. In the second approach, 1-g modal spectra at a range of known fill levels are used in a cross-correlation calculation to predict fill levels associated with a zero-g modal spectrum. Flight data for both approaches are consistent with finite element predictions using a simple fluid-structure interaction model. In both settled and unsettled microgravity environments, MPG meets or exceeds NASA Roadmap goals

Keywords for in-space propellant mass gauging.

Artemis Program • Propellant Gauging • Microgravity Liquids • Propellant Depot • Lunar Gateway • Modal Analysis

\section{INTRODUCTION}

NASA's proposed Lunar Gateway (LG) represents a compromise between the limited delta- $\mathrm{V}$ achievable by the Orion Multipurpose Crew Vehicle (MPCV) and the programmatic goal of a sustainable human presence on and near the lunar surface. The MPCV comprises the Crew Capsule (CC) and its European Service Module (ESM). The propellant load and mass of the CC/ESM architecture, designed as part of the Constellation program, preclude the spacecraft from reaching and returning from a low lunar orbit (Whitley and Martinez, 2016). Instead, the MPCV will dock at the LG, located in a halo orbit near the Earth-Moon Lagrange Point 2 (EML-2). The LG will provide staging facilities for lunar landers and ascent vehicles.

The first element of the proposed LG architecture is the power and propulsion element (PPE). The PPE provides station-keeping and orbit-raising functions as well as chemical and electric (xenon) propellant refueling. Sustainable human presence on the surface of the moon and, ultimately, human missions to Mars, depend critically on the ability to provide in-space propellant transfer and propellant depot functions to visiting spacecraft. The essential importance of providing inspace propellant transfer is one of the reasons that the PPE was one of the first Gateway elements to be contracted. The risk reduction and economic potential of deep space exploration depend on the existence of high-TRL on-orbit propellant transfer (Notardonato et al., 2012).

In-space liquid propellant transfer, however, has been achieved only in small-scale demonstration tests of limited scope. End-to-end propellant transfer experiments on flight hardware have not yet been conducted. For this reason, in-space propellant transfer remains a "critical path" objective for fuel depot capability (Casanova et al., 2017). The primary challenge of in-space liquid propellant transfer is low-gravity mass gauging of liquids and liquid distribution knowledge inside propellant tanks. Despite over 50 years of work in this area, accurate, low-g liquid mass gauging remains an unsolved problem as well as a problem of significance to a variety of potential customers beyond the human spaceflight community (Holmes et al., 2019). The critical nature of low-g gauging is captured in the 2020 NASA Technology Taxonomy (NASA, 2020).

The Modal Propellant Gauging (MPG) experiment is one of several emerging approaches to low-gravity liquid propellant gauging. MPG provides real-time, noninvasive (no tank penetrations), gauging of both cryogenic and Earth-storable

\footnotetext{
Corresponding author: Kevin M. Crosby

E-mail: kcrosby@carthage.edu
} 
propellants by measuring the "added-mass" effect of contained liquid on the resonant mode frequencies of thin-walled tanks subject to acoustic excitation.

MPG has been demonstrated in Earth gravity (1-g) on a variety of subscale and flight hardware propellant tanks, including a hot-fire test of the Methane-LOX system onboard the Morpheus Prototype Lander, pressure and thermal cycle tests on a Space Shuttle OMS tank, the Orion ESM propellant tank (qualification unit), and various composite tanks with both cryogenic propellant simulants and water (Crosby et al., 2019). MPG has also been extensively tested on parabolic flights, across three different flight campaigns (Crosby et al., 2016).

In this report, we share the results of two suborbital flight tests of the MPG technology on the Blue Origin New Shepard (NS) crew capsule. The payload experiments were conducted aboard the NS P7 and NS P9 (payload) missions in January 2019 and December 2019, respectively. Both flights carried the same hardware and carried out essentially the same tests, with the first flight considered a "shakeout" test to determine the functionality of the full system hardware and to record environmental test data for use in the second flight, which acquired the full set of MPG data reported here.

\section{SURVEY OF LOW-GRAVITY PROPELLANT GAUGING TECHNOLOGIES}

Low-gravity propellant gauging technologies in wide use include those that depend on bookkeeping, equations of state, and thermal mass estimation. Bookkeeping methods employs flow rate monitors or, often, just assumptions about flow rates during thruster firings to estimate the total amount of propellant consumed over time. Such methods accumulate error over time and therefore introduce growing uncertainties in the estimate of remaining propellant mass and resulting estimation of end-of-life (EOL) (Dodge, 2008). Here, "uncertainty" in remaining propellant refers to the estimated percent of uncertainty between actual and indicated propellant.

Equation of state (PVT) methods rely on the inference of the liquid volume $(\mathrm{V})$ fraction within a tank pressurized by an external pressurizing gas (usually, helium) based on measurements of pressure $(P)$ and temperature $(T)$ and the application of an appropriate equation of state to the ullage gas. In the PVT approach, the tank is considered isothermal, and accuracy depends in part on the validity of this assumption. As in the case of bookkeeping methods, propellant mass estimates derived from applications of PVT methods are increasingly inaccurate at lower fill fractions, resulting in reduced accuracy near EOL (Yendler, 2006). Mass gauging uncertainty using PVT and bookkeeping methods varies widely with the type of spacecraft, with literature estimates of gauging accuracy near EOL ranging from 3.5\% to $10 \%$ or more.

Thermal mass estimates rely on measurements of the temperature change induced in a propellant tank by external heating. Thermal response is compared to simulation results based on a tank-specific thermal capacitance model computed over a range of fill fractions. The uncertainty of propellant mass estimates based on thermal propellant gauging (TPG) methods depends sensitively on the sophistication and fidelity of the tank thermal model (Yendler, 2006).

In addition to low-gravity methods, settled propellant levels may be estimated using capacitance probes and impedance sensors. In microgravity, capillary forces drive liquid propellant along the surfaces of such probes rendering their measurements useless. These electrical measurements are performed on settled liquids and are therefore used only during thrusting maneuvers and are subject to error induced by residual adhered liquid on sensors.

Recent advances in gauging using radio frequency (RF) probes to correlate electromagnetic cavity modes with $1-\mathrm{g}$ library data have demonstrated $1 \%$ gauging accuracies in the laboratory for settled propellants but require additional hardware and invasive tank modifications (Zimmerli et al., 2011).

A complete review of extant gauging technologies, their flight heritage, and their limitations is provided in the work of Dodge (2008). Each family of methods has relatively reduced accuracy at low tank fill fractions, and, therefore, propellant gauging is the primary limiting factor in the estimation of mission EOL. Error estimates on most of the gauging methods range from $1 \%$ to $20 \%$ or more. For example, errors in the computed estimate of consumed propellant through the bookkeeping methods are typically around $\pm 3.0 \%$ (but much higher near EOL). Adding propellant mass to account for gauging errors can result in significant launch costs and/or reduced mission life.

In MPG, the gauging errors in settled low-gravity propellant are relatively well characterized by the material properties of the tank wall and liquid propellant; modal frequencies depend only on tank geometry, material properties, and liquid density. In the MPG approach, slosh affects instantaneous gauging accuracy but can be "averaged over" for slosh dynamics that are roughly periodic. Computational overhead in MPG is minimal; a development path for radiation-hardened deepspace MPG avionics has been established by an avionics team at the Johnson Space Center.

\section{OVERVIEW OF THE MPG APPROACH}

The MPG technology concept exploits the "added-mass" effect of liquid adhered to surfaces, such as the tank wall in 


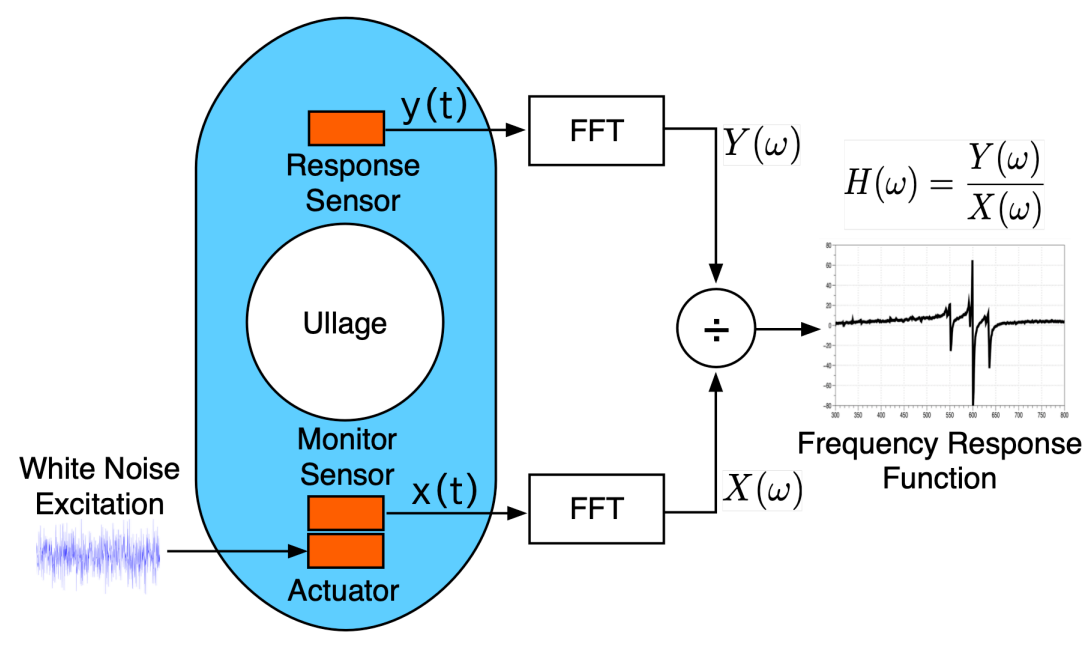

Figure 1. The MPG concept. Surface waves excited on the tank wall induce acoustic resonances with frequencies that depend sensitively on the mass of liquid adhered to the wall. Modal analysis is used to detect mode shifts. MPG: Modal Propellant Gauging.

a propellant system. The presence of liquid in contact with a solid surface results in a higher effective mass of the surface, revealed through a reduction in the modal frequencies associated with the surface. Any fluid mass loads surfaces with which it is in contact. The mass-loading effect is in proportion to the contact area and the density of the fluid. MPG implements standard modal analysis in a real-time computational scheme to detect these modal shifts and translate them into estimates of contained volume. The MPG concept is illustrated in Figure 1.

The surface of the tank wall is subjected to lowamplitude, broadband acoustic white noise through a thin, flexible patch transducer adhered to the tank surface. This "actuator" excites resonances in the tank wall that are affected by the presence of liquid adhered to the surface through the added mass effect. Identical piezoelectric (PZT) patch transducers both near and far from the actuator detect the response to the white noise excitation. Sensor outputs are digitized and converted to frequency domain data through Fast Fourier Transforms (FFT). The FFT of the sensor signal from nearby the actuation point carries the broadband white noise frequency content, which is essentially a constant $X(\omega)=S_{0}$ in the frequency domain, where $\omega$ is the angular frequency. $N$ sensors, $i=1, \ldots, N$ located far from the actuation point, carry frequency content that includes both the white noise signal and the characteristic modal response of the tank wall, $Y_{i}(\omega)=S_{0} Y_{R, i}(\omega)$, where $Y_{R, i}$ represents the modal response of the $i$-th sensor. The frequency response function (FRF) is the ratio of the FFT from sensor locations far from the actuator to the FFT of sensor response near the actuator,
$H_{i}(\omega)=\frac{Y_{i}(\omega)}{X(\omega)}$

The FRF $H_{i}(\omega)$ contains the frequency response of the tank wall with the excitation signal (common to both sensors) effectively nulled out.

While FRFs are, in general, complex and multimodal, the frequency response of most structures is fairly simple at low frequencies near the fundamental modes, where the structure can be approximated as a single-DOF oscillator. It is these modes that are also most sensitive to liquid mass loading and are therefore of the greatest interest for MPG. Typical modal response of a cylindrical tank under broadband acoustic excitation is shown in Figure 2 for a pair $(N=2)$ of sensors. The low-lying modes are well-separated and are sharply defined in the thin-walled metallic tank. In this case, the liquid propellant simulant is water and the tank is a 7.6 I carbon-overwrapped pressure vessel (COPV).

The authors have developed a number of algorithms for translating modal response in liquid-filled tanks into estimates of contained liquid volume (Crosby 2018; Crosby et al., 2019, 2020). Here we describe the cross-correlation approach to modal gauging. In the cross-correlation method, the modal response of a tank for which the propellant level is desired is compared to a library of modal data of that tank at closely spaced fill levels to identify a "best-match" modal spectrum. The known fill level of the best-match spectrum is then associated with the modal response of the unknown fill-level. The "best-match" spectrum is the one for which the cross-correlation between the unknown modal spectrum and 


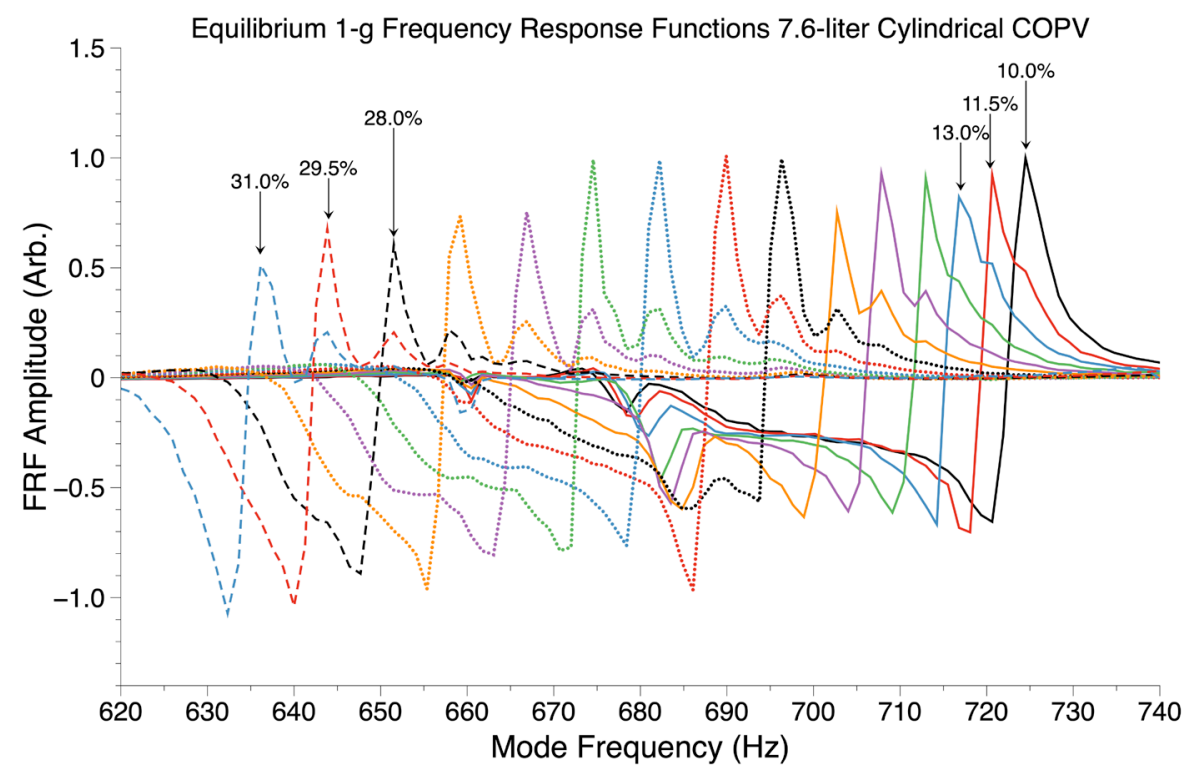

Figure 2. Typical modes in a subscale cylindrical tank at liquid fill levels between $10 \%$ and $31 \%$ of full-tank volume. A frequency resolution of $1-\mathrm{Hz}$ results in gauging resolutions of below $1.0 \%$.

the known modal spectrum is maximal as measured by the Pearson correlation coefficients (Press et al., 1992).

The cross-correlation approach requires that we acquire the modal response of the tank across all desired fill-levels. $A$ priori, there is no expectation that the zero-g modal response of the tank will be correlated well with the library data obtained in 1-g. However, early indications from parabolic and suborbital flights discussed in the following paragraphs suggest that the correlation is quite good, and that $1-\mathrm{g}$ reference data may be used to predict zero-g fill levels.

As a practical example, a cross-correlation method, in which real-time modal response is compared to a library of modal data to identify a "best-match" modal spectrum, has been applied to the Orion ESM tank, during qualification testing at the Airbus facility in Bremen, Germany. In these tests, the tank was repeatedly filled with water and drained from nearly full to empty while pressure was maintained at various levels between 1.0 and 10.0 bar. The drain rates during the tests were much lower than would occur in use, with typical drain rates between $5.0 \mathrm{~kg} / \mathrm{min}$ and $60 \mathrm{~kg} / \mathrm{min}$.

In Figure 3, a typical pressure cycle of the Orion ESM tank is shown with mass measurements provided by the facility (solid curve) and MPG predictions for the mass based on modal response during the pressure cycle. The reference modal data was used to generate the "library" modal data and were obtained in a separate pressure cycle conducted at a different drain rate than the test run. MPG achieves an average gauging accuracy of $0.24 \%$ during all pressure cycles of the qualification tank. Gauging accuracy here is defined as the percent difference between the MPG-predicted fill volume (or mass) and the known fill volume (or mass) for the test.

These tests were, of course, in 1-g and under slow fill and drain rates compared to flight conditions. To assess the viability of the MPG approach for propellant transfer and fuel depot applications, it is necessary to perform flight tests in microgravity and with appropriately scaled drain rates. The authors have conducted a series of parabolic flight campaigns to assess the efficacy of MPG for unsettled, sloshing liquids in microgravity (Crosby et al., 2016). The parabola duration is too short to achieve equilibrated liquid surfaces in tanks of even modest scale, so the parabolic flight data represent only the extreme case of slosh-driven flow. Results from the parabolic flight tests suggest gauging accuracy of 3-5\% during microgravity sloshing (Crosby et al., 2016).

To achieve equilibrated liquid surfaces in microgravity in subscale tanks of reasonable size, at least 1-2 min of clean microgravity is required. The flight profile of the NS vehicle meets this criterion with a nominal $200 \mathrm{~s}$ of extremely clean (sensed acceleration $\sim 0.001 \mathrm{~g}$ ) microgravity between CC separation from the booster and the onset of atmospheric drag during re-entry.

\section{EXPERIMENT OBJECTIVES}

The NS flight test of MPG had two central objectives. The first objective was to prove a key hypothesis of the MPG approach, which is that the mass-loading effect of 


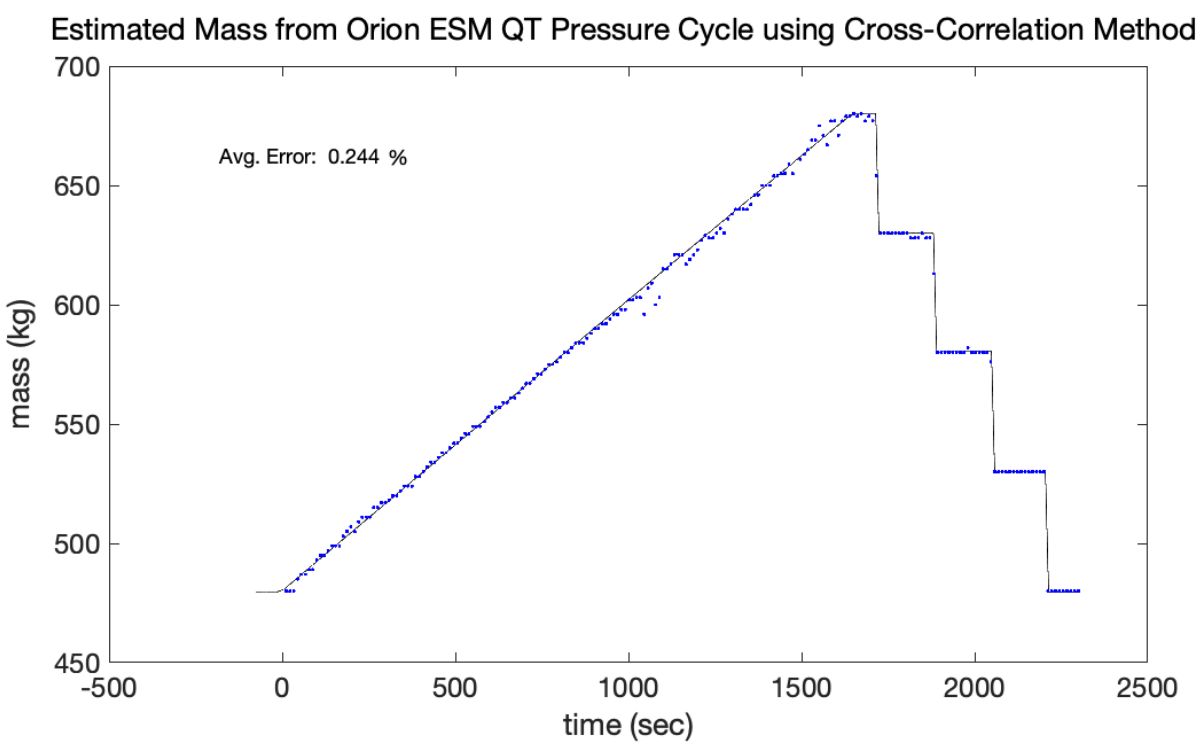

Figure 3. MPG mass measurements on the Orion ESM qualification tank. The point data are instantaneous mass estimates produced by MPG using the cross-correlation method during a drain cycle. The solid line is the facility mass data during the drain. ESM: European service module; MPG: Modal Propellant Gauging.

liquid adhered to the inner walls of a propellant tank in an equilibrium low-gravity surface configuration is uniquely related to the mass of liquid present in the tank. Establishing this relationship is critical to the viability of the proposed technique and can only be established under long-duration microgravity experiments. The second objective is to validate analytical and numerical models of modal response in thinwalled tanks. These models are discussed in the Flight Data and Analysis section.

\section{PAYLOAD EXPERIMENT}

The MPG suborbital payload experiment conforms to the NS "Double Locker" form factor. The rig is shown in Figure 4 and consists of three transparent polycarbonate cylindrical tanks that are filled to different levels with water. Each tank is 6 -in. in diameter and 10-in tall. The water is dyed with different colors in each tank to aid the researchers in distinguishing tankspecific liquid equilibration dynamics.

The tank geometry for the NS experiment was chosen as a compromise between the payload volume constraints of the NS Double Locker and the desire to have realistic liquid behavior in microgravity. The tank diameter was selected to be as large as possible given the volume constraints to provide for reasonable slosh and equilibration dynamics of the water. Water is essentially nonwetting on polycarbonate (contact angle of $\sim 80^{\circ}$ ), which complicates the development of full-fidelity tank modeling that is both small enough to fit in the vehicle but has large enough diameter to demonstrate realistic dynamic behavior with water.

The zero-g equilibration time is the time required for the liquid mass to find its zero-g equilibrium distribution in the tank after Main Engine Cut-Off (MECO) and Crew Capsule Separation (CCSEP), when the payload transitions from 1-g to 0 -g. In cylindrical tanks, this equilibration time is predicted to scale with the diameter $D$ of the tank as $\sqrt{D}$ (Ibrahim, 2005). Our computational fluid dynamics (CFD) experiments, discussed in the Flight Data Analysis section, suggest that the equilibration time in our subscale tanks should be approximately $30 \mathrm{~s}$, and indeed the video from the first NS flight, shows that the liquid, scrambled upon CCSEP, does find its final, equilibrated configuration in around $25 \mathrm{~s}$ (MPG P9 Flight Video, 2019). Figure 5 shows the three tanks at $25 \mathrm{~s}$ after CCSEP in the NS P9 mission. Frame-by-frame analysis shows that, for each tank, the liquid-air interface has no component of velocity in the direction along the long axis of the tanks greater than $\sim 1 \mathrm{~cm} / \mathrm{s}$.

Three PZT patch transducers on each tank serve as the actuator and response sensors. The actuators on each tank are driven by a piezo amplifier that multiplies the output of a white noise generator to a $100 \mathrm{~V} p-p$ signal. The excitation signal is a random amplitude signal across the frequency range $50-5000 \mathrm{~Hz}$. When empty, the tanks have fundamental excitation resonances at around $400 \mathrm{~Hz}$.

Data acquisition is performed by a high-speed analogto-digital (ADC) converter that samples and stores data 28.8 kSamples/s, which is well above the Nyquist Frequency for 


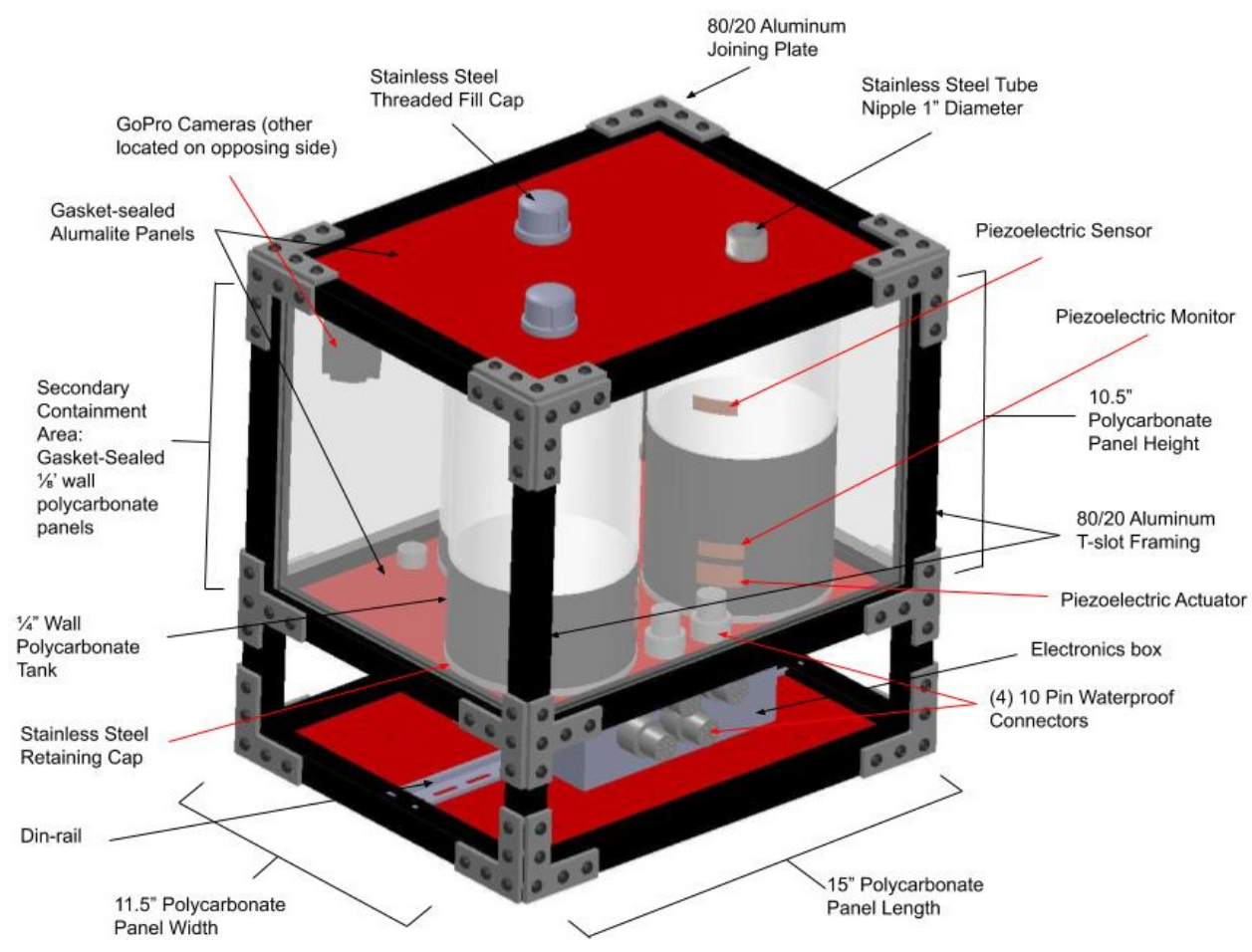

Figure 4. NS payload experiment rendering. Three transparent, cylindrical tanks contain water at different fill levels. MPG sensors and data acquisition system record the modal response of each tank while cameras record the liquid motion during the flight. MPG: Modal Propellant Gauging; NS: New Shepard.

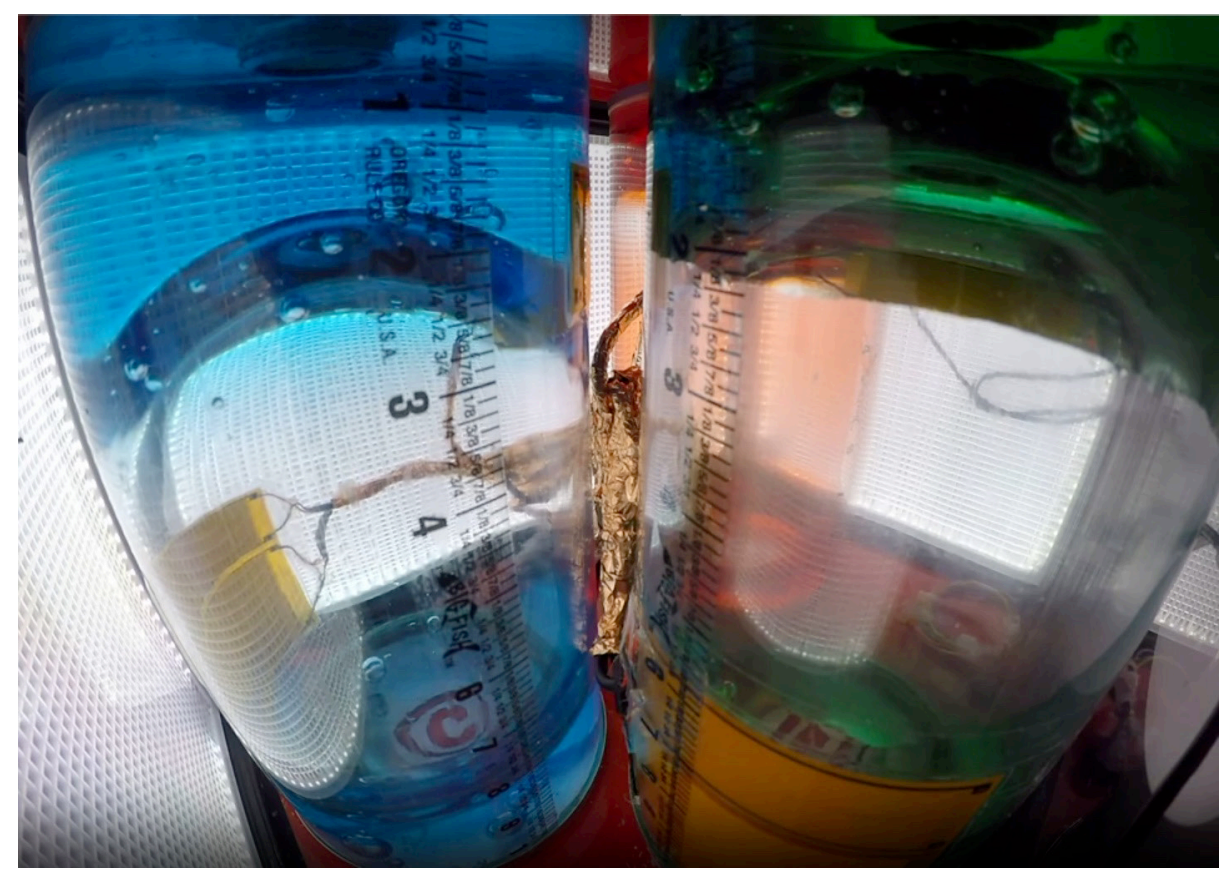

Figure 5. Tanks 3 (Blue) and 1 (Green) imaged 25 s after CCSEP when surface interface motion along the tank wall had essentially stopped. The liquid in each tank, scrambled by the impulse of CCSEP, reached an equilibrium state with roughly half of the volume adhered to the top and half to the bottom of each tank. CCSEP: Crew Capsule Separation. 


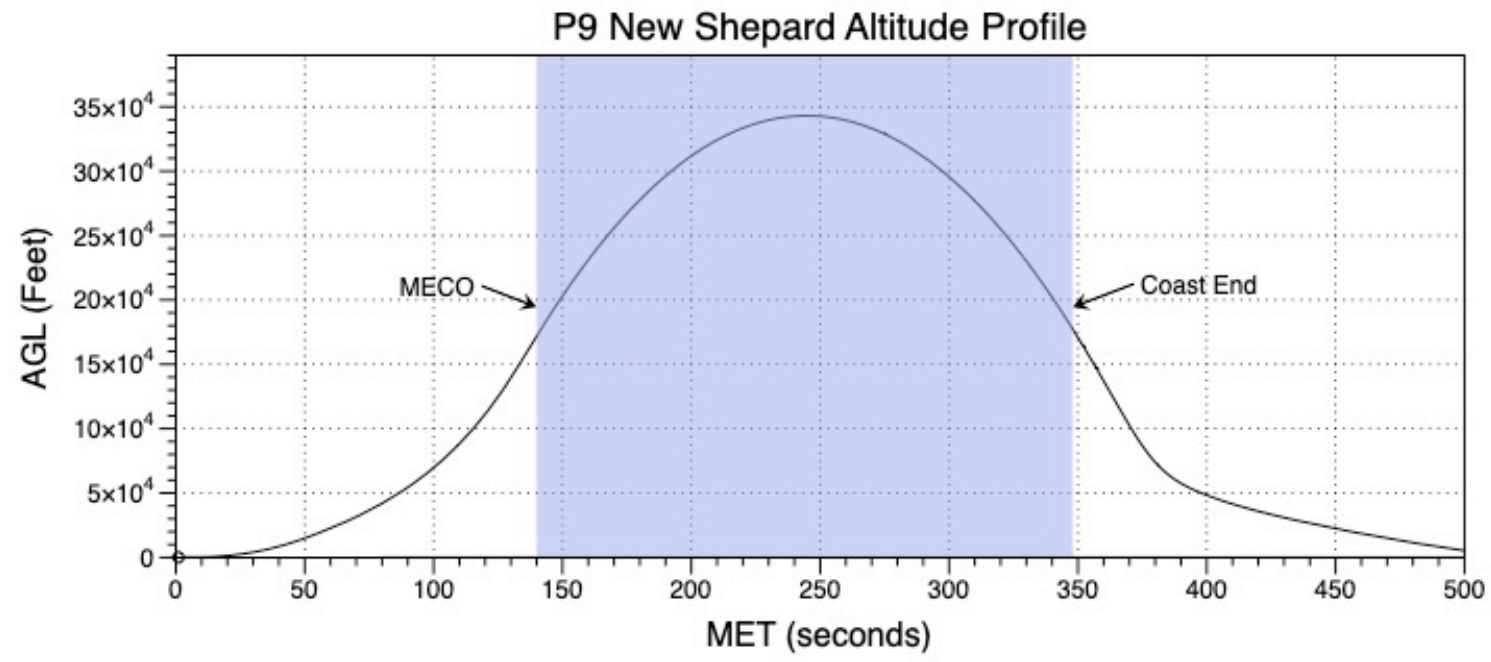

Figure 6. The NS flight profile. Altitude above ground level (AGL) vs. mission elapsed time (MET) is shown for the P9 mission. Courtesy: Blue Origin. NS: New Shepard

the modal signals of interest $(190-450 \mathrm{~Hz})$. The ADC samples sensor output continuously from launch through the "coast end" phase of the mission, recording raw time series data from each of the two sensors on each of the three tanks.

Two cameras positioned to capture the liquid position in each tank record continuously throughout the flight. The camera data are used to correlate liquid surface position and distribution with the modal response of the tank at each of the three fill levels.

The tanks and cameras are enclosed in a secondary containment vessel with diffuse LED light panels on three sides for illuminating the tanks during flight.

\section{FLIGHT PROFILE}

The P9 NS flight profile, shown in Figure 6, provides approximately 200 s of microgravity between MECO at $L+140 \mathrm{~s}$ and Coast-End at $L+348 \mathrm{~s}$, with a transient impulse from CCSEP at $L+160 \mathrm{~s}$. The MPG experiment acquired modal data from $L+0 s$ through $L+280 s$ and video data between $L+65 \mathrm{~s}$ and Coast-End. The maximum sensed acceleration during launch was 9.6-g along the axial direction and apogee for this mission was $343,000 \mathrm{ft}$.

\section{Flight Data and Analysis}

Flight data for the MPG missions consist of raw time-series acoustic data acquired from two sensors on each of the three tanks as well as video data of the liquid behavior in the tanks acquired from the cameras mounted inside the secondary containment vessel. These data are used to address the study objectives identified in the Experiment Objectives section of this report. Specifically, the raw sensor data are used to generate FRFs from which primary resonant modes for each tank are extracted. These mode frequencies are compared with analytical predictions, finite element (FE) calculations, and experimental data from laboratory testing under Earth gravity. Video data are used to correlate modal responses with liquid surface location within the tanks.

\section{CFD Model}

The CFD simulations were carried out using OpenFoam and the interFoam solver (OpenFoam, 2020). InterFoam implements the volume of fluid (VOF) method to track the freesurface interface between two immiscible, isothermal, and incompressible fluids in the absence of turbulence (Hirt and Nichols, 1981). In our CFD simulations, the simulation volume is a right circular cylinder with rigid boundaries corresponding to the interior dimensions of the transparent polycarbonate cylinders used in the NS experiment. A grid convergence experiment was conducted to determine the minimum node count for the simulations. For most of the simulations carried out for equilibration time studies, 20,000 spatial nodes were used in the geometry discretization. The CFD calculation was carried out in 20 fill increments between $1 \%$ and $50 \%$ of the full-tank volume, including each of the three fill levels for the three tanks flown in the P9 NS flight.

To reproduce the impulse delivered to the liquid by the CCSEP from the booster just prior to the onset of weightlessness, the initial conditions for the CFD calculations correspond to a spherical volume of water initially centered in the tank volume with a speed of $0.1 \mathrm{~m} / \mathrm{s}$ directed toward one of the two flat faces. Neither the direction of the initial velocity vector for the liquid mass nor its magnitude influences 
Time: 30.02

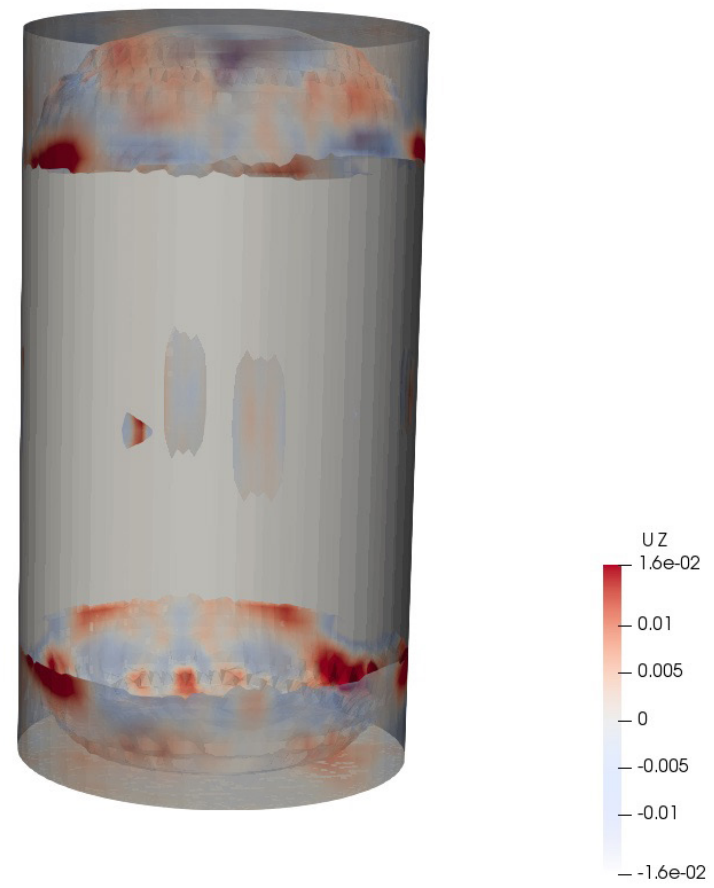

Figure 7. Representative equilibrated, zero-g liquid distribution from CFD simulations of the experiment tank at $\mathrm{T}+30 \mathrm{~s}$ after initial impulse. Fill fraction is $12 \%$ of full-tank volume (524 ml). CFD: Computational fluid dynamics.

the final equilibrated state of the liquid volume in zero-g. The impulse choice was dictated by the maximum speed that is consistent with the grid discretization choice and the desired maximum Courant Number. A contact angle of $80^{\circ}$ between the polycarbonate tank wall and the water "propellant" is assumed in the CFD. A representative CFD liquid distribution is shown in Figure 7. The simulations were terminated when the magnitude of the velocity of the fastest fluid parcel in the simulation was $<1 \mathrm{~cm} / \mathrm{s}$. We considered this time to be the equilibration time for the liquid and is typically on the order of 20-30 s. Each CFD fill-level calculation required roughly $2 \mathrm{~h}$ of computation time on an 8-core AMD Epyc 7252 Processor at $3.1 \mathrm{GHz}$ with $64 \mathrm{~GB}$ RAM.

In weightless conditions, the water in the tank equilibrates in roughly equal size domains at the top and the bottom of the tank. This is assured by the interior corners and high curvature of the tank walls at the top and bottom. Video data from two flights and CFD modeling confirm this behavior.

\section{Finite Element Model}

The simplicity of the tank geometry in the NS flights permits a relatively accurate estimate of mode shapes and mode frequencies for the empty tank from FE methods. To model the influence of the liquid mass on the modal response of the tank, the FE calculations were carried out according to the following scheme. First, CFD estimates of the mass and shapes of the zero-g equilibrium liquid distributions were used to create mesh models of the liquid distributions at each fill fraction. These meshes were exported in Stereolithography/ Standard Triangle Language (STL) format and used to build FE models of the tank at various fill levels.

The FE modeling was carried out using the known tank material properties and geometry with a "distributed mass" of equivalent water defined by the CFD-generated STL meshes at the top and bottom of each tank. In this way, high-fidelity zero-g estimates of modal frequencies were obtained at each fill fraction studied in the CFD model. The FE calculations were conducted using SolidWorks Simulation FE Analysis module and its Distributed Mass function (Dassault Systems, 2019).

It is interesting to note that, while the CFD-driven FE calculations provide a predictive model of the distributed mass effect of the liquid on the tank resonance modes, reasonably accurate estimates of modal frequencies in zero-g can be obtained from simpler FE calculations of the liquid described by a single equivalent mass located at one end of the tank with a planar interface between air and liquid. Indeed, the agreement between 1-g laboratory experiments on the tank and the zero-g modal data is within $1 \%$ at the three fill levels for which we have zero-g modal data. This 


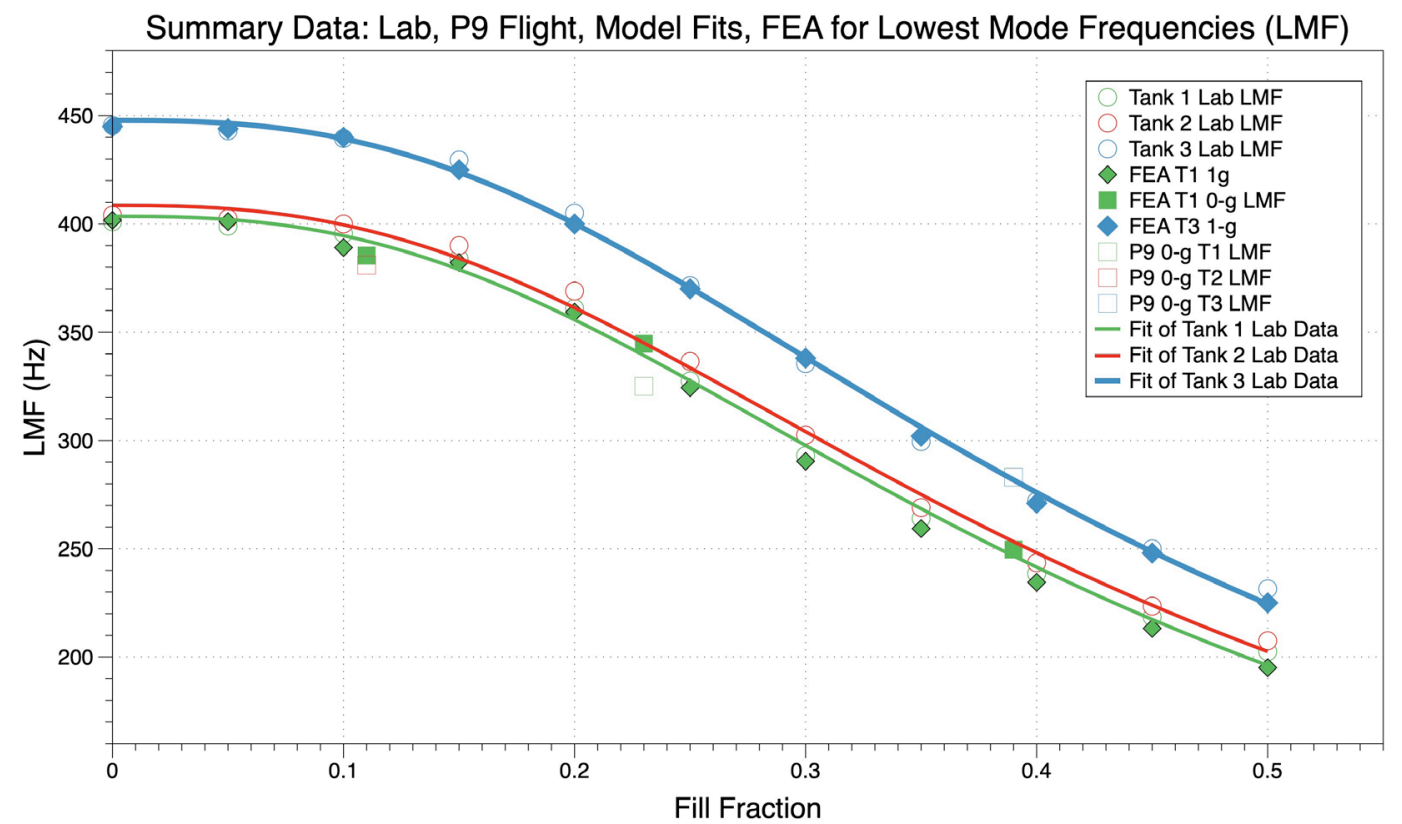

Figure 8. Summary data for FE calculations, 1-g laboratory data, and P9 flight data. LMF as a function of fill fraction. The solid curves are 2-parameter best-fit lines with functional form described by Eq. (2). The fit parameters are $A=19.3$ and $b=2.69$. FE: Finite element; LMF: Lowest mode frequency.

result is suggestive that 1 -g laboratory and/or FE calculations can be used to generate reasonably accurate estimates of 0 -g modal response.

While the current MPG suborbital experiment allowed only three static fill levels, in the laboratory, we are able to make modal measurements with arbitrary fill-fraction resolution by filling and draining the tanks in small increments. In Figure 8 , we show a summary of the lowest mode frequency (LMF) data for 1-g laboratory fills, FE model predictions, and the NS flight data. The 1-g laboratory data are plotted against the fill fraction at intervals of $5 \%$ of the full-tank volume for each of the three tanks. The NS flight data are shown only for the equilibrated portion of the flight profile (after the liquid surface had found its zero-g equilibrium configuration at $L+190 \mathrm{~s}$ ). While Tanks 1 and 2 in the NS experiment have similar modal response, Tank 3 (blue-colored data in Figure 8) has higher average modal frequencies than the other two tanks. We attribute this difference to differences in construction technique that led to a slightly lower mass for Tank 3 (less epoxy was used in the bonding of components), leading to a $2 \%$ lower empty weight for Tank 3 relative to Tanks 1 and 2 .

The "best-fit" curves (solid lines in Figure 8) are calculated from a fit function of the form

$$
f_{\text {fit }}=\frac{f_{0}}{\sqrt{1+A r^{b}}}
$$

where $f_{0}$ is the empty-tank LMF, $r$ is the fill fraction, and $A$ and $b$ are fit parameters. The form of the best-fit function was chosen to generalize the analytical result of Chu, who calculated (approximately) the natural mode frequencies of a vibrating circular cylindrical tank with rigid plate boundaries and partially filled with liquid (Chu, 1963). Using the so-called Reissner approximation to the Donnell-Yu shell equations, an approximate expression for the frequency $f_{m n}$ corresponding to a tank vibrating with longitudinal mode $n$ and circumferential mode $m$ is given by

$$
f_{m n}=\frac{f_{m n}^{0}}{\sqrt{1+M^{*} \frac{\rho_{f} R}{\rho_{0} t}}}
$$

where $f_{m n}^{0}$ is the empty-tank modal frequency of the $(m, n)$ mode, $\rho_{f}$ is the liquid density, $\rho_{0}$ is the tank material density, $R$ and $t$ are the radius and thickness of the cylinder, and $M^{*}$ is the ratio of the added mass, due to the liquid, to the tank empty mass (Ibrahim, 2005). Eq. (3) is an approximate solution for the breathing mode frequencies and assumes uncoupled radial and transverse shell vibrations and a fluid-structure interaction in which the liquid-loaded tank has essentially the same dominant breathing mode as the empty tank. Eq. (3) agrees with measured frequencies at all liquid fill levels to within $8 \%$ for the $n=1$ breathing mode of the cylinder (Ibrahim, 2005). 
Table 1. Cross-correlation-predicted fill fraction and NS P9 known fill fractions. \% Error is calculated from $100 \times$ [Predicted Fill Volume - Known Fill Volume|/Total Tank Volume. The total tank volume is $4356 \mathrm{ml}$.

\begin{tabular}{ccccc}
\hline Tank & $\begin{array}{c}\text { Fill volume } \\
(\mathbf{m l})\end{array}$ & Fill fraction & $\begin{array}{c}\text { MPG CC } \\
\text { predicted fill } \\
\text { volume }(\mathbf{m l})\end{array}$ & $\begin{array}{c}\text { \% Error in CC } \\
\text { predicted fill } \\
\text { volume (\%) }\end{array}$ \\
\hline Tank 1 & 1000 & 0.230 & 1025 & $0.57 \%$ \\
Tank 2 & 500 & 0.115 & 540 & $0.92 \%$ \\
Tank 3 & 1500 & 0.344 & 1485 & $0.34 \%$ \\
\hline
\end{tabular}

CC: Crew capsule; MPG: Modal propellant gauging.

Fahy and Gardonio provided a generalized expression for the modal frequencies of a partially filled structures that is identical to Eq. (3) but with $M^{*}$ replaced with the ratio of the generalized modal mass of the fluid-loaded structure to the modal mass of the fluid-free structure (Fahy and Gardonio, 2007). In general, the fluid-loaded modal mass is not linear in the fluid mass, so our fit parameter $b$ is not constrained to be 1 as it is in the approximate expression for the frequencies of the fluid-loaded tank given by Eq. (3). The fit to the 1-g laboratory data shown in Figure 8 requires $b=2.69$.

The data in Figure 8 suggest that 1-g laboratory modal analysis and FE calculations can be used to predict the LMF of equilibrated zero-g fill-levels in the simple cylindrical tanks considered here. However, to accurately estimate the fill fraction from modal data at fill fractions below around 0.1, the LMF is insufficient. At both low and high fill fractions, the LMF is relatively insensitive to variations in fill fraction, as indicated by the flattening of the curves in Figure 8 as the fill fraction approaches zero. This is a general feature of the modal response of fluid-loaded structures (Fahy and Gardonio, 2007).

It is still possible to achieve sub-1\% gauging accuracy at extreme fill fractions by applying the cross-correlation matching method discussed above in the context of the Orion ESM tank tests. In the cross-correlation approach, the modal response across a range of frequencies is matched against a library of modal responses at various fill fractions. Correlation coefficients are computed for each pairing of modal spectra and the library response with the highest correlation coefficient (and a $p$-value of $<0.005$ ) is selected as the match for the modal spectrum for which a fill fraction is desired. The fill fraction of the library response is then assigned to the unknown spectrum.

In the case of the Orion ESM tank, continuous modal responses were acquired across the fill range, allowing arbitrarily closely spaced fill levels to be stored as reference data for use in the cross-correlation analysis. For the NS experiment, each of the tanks was filled at $\sim 5 \%$ fill intervals from empty to full to obtain a library of modal responses. This was achieved by repeatedly adding $200-250 \mathrm{ml}$ to each tank and measuring the 1-g modal response at each fill level. The 0 -g predicted and known fill fractions for each tank is shown in Table 1.

\section{The MPG-PROTO Experiment}

The NS experiments flown on P7 and P9 were limited to three static fill levels in relatively unrealistic tank geometries with no propellant management devices present. In contrast, the parabolic flight experiments developed for MPG have a flow loop system that permits the metered movement of liquid between multiple experimental tanks in both high-g and low-g conditions. The next iteration of the suborbital flight experiment series will capitalize on the team's parabolic flight experiment history to develop a suborbital flight experiment that implements a high-fidelity subscale spherical tank modeled after the primary propellant tank on the LG program's Power and Propulsion Element (PPE). The Propellant Refueling and On-orbit Transfer Operations (PROTO) experiment features a tank with a radial vane-and-sponge style PMD, vent and pressurization ports, a flow-loop that continuously drains and fills the primary tank through a pump-driven flow-loop connected to a reservoir tank at a rate of approximately 4 LPM. The flight experiment is scheduled to fly on NS P11.

The PROTO experiment will bridge the gap between the equilibrated zero-g static fill-level data obtained in the P7 and P9 missions with the 1-g dynamic drain data obtained in the laboratory on full-scale flight hardware and the sloshing zero-g data obtained on parabolic flights. With PROTO, MPG will close the loop on fully autonomous modal gauging by applying real-time cross-correlation methods to estimate propellant levels during both static fill and continuous drain operations.

\section{SUMMARY AND CONCLUSIONS}

The MPG project has demonstrated sub- $1 \%$ gauging accuracy in flight hardware under 1-g conditions and has demonstrated $1-3 \%$ gauging accuracy in subscale zero-g testing of sloshing liquids aboard parabolic flights. In the tests reported here, the first equilibrated zero-g MPG results are demonstrated to agree with both FE predictions and 1-g laboratory data to within $1 \%$. Liquid surface distributions in cylindrical subscale tanks are shown to agree with CFD calculations and equilibration times for the experimental tanks are also comparable to CFD estimates ( $2 \%$ relative difference).

The NASA technology roadmaps identify a target for zero-g propellant gauging errors of $1 \%$ of the full-tank volume for settled or equilibrated propellant and 3\% for unsettled propellant (NASA, 2015). Extant methods of gauging such as Bookkeeping and PVT have zero-g gauging errors that 
Table 2. Summary of MPG tests and results.

\begin{tabular}{|c|c|c|c|c|c|c|}
\hline Experiment & Hardware & Platform & Test conditions & Gravity level & $\begin{array}{l}\text { \% Error in } \\
\text { LMF }\end{array}$ & $\begin{array}{l}\% \text { Error in CC predicted fill } \\
\text { volume (\%) }\end{array}$ \\
\hline \multirow[t]{2}{*}{ MPG-I-III } & Subscale Pill-shaped & Lab & Settled static & $1-g$ & $<1 \%$ & $0.25 \%$ \\
\hline & $\begin{array}{l}\text { polycarbonate tanks } \\
\text { polyansparent }\end{array}$ & Parabolic & Sloshing & $0-g$ & $3-5 \%$ & - \\
\hline KSC Cold-Tank test & Shuttle OMS tank & Lab & $\begin{array}{l}\text { Settled slow drain } \\
\quad \text { and fill }\end{array}$ & $1-g$ & - & $<0.5 \%$ \\
\hline $\begin{array}{l}\text { Airbus Orion ESM } \\
\text { tank qualification }\end{array}$ & $\begin{array}{c}\text { Orion ESM qualification } \\
\text { tank }\end{array}$ & Lab & $\begin{array}{l}\text { Settled slow drain } \\
\quad \text { and fill }\end{array}$ & $1-g$ & $<1 \%$ & $0.24 \%$ \\
\hline Morpheus hot-fire & $\begin{array}{l}\text { 48-in diameter spherical } \\
\text { propellant tanks }\end{array}$ & $\begin{array}{c}\text { Morpheus } \\
\text { Prototype } \\
\text { Lander }\end{array}$ & $\begin{array}{l}\text { 30-s vacuum hot- } \\
\text { fire at the NASA } \\
\text { Plumbrook Station } \\
\text { B-1 Chamber }\end{array}$ & $1-g$ & - & $0.12 \%$ \\
\hline P9 Mission & $\begin{array}{l}\text { Subscale polycarbonate } \\
\text { cylindrical tanks }\end{array}$ & NS & P9 Suborbital Flight & $0-\mathrm{g}$ & $<1 \%$ & $<1 \%$ \\
\hline
\end{tabular}

CC: Crew capsule; COPV: Carbon-overwrapped pressure vessel; ESM: European Service Module; LMF: Lowest mode frequency; MPG:

Modal propellant gauging; NS: New Shepard.

can exceed $10 \%$ at low fill fractions. The NS P9 mission data confirm that MPG meets and exceeds the equilibrated propellant gauging objective. MPG has been tested on several tank systems and in a variety of both $1-\mathrm{g}$ and $0-\mathrm{g}$ environments. A summary of these experiments and results is provided in Table 2.

These results suggest that MPG is a viable approach to dynamic, low-gravity mass gauging of liquids, including propellants and life-support fluids in microgravity environments. Currently, MPG is the only propellantagnostic and noninvasive low-gravity gauging technology in development. Forward challenges for the technology include assessing the degree to which $1-\mathrm{g}$ modal data can be used to predict $0-\mathrm{g}$ fill levels in a full-scale tank, the development of avionics to meet the power and mass requirements of the 2020 NASA Technology Taxonomy, and a rigorous assessment of MPG under 0-g propellant transfer and engine burns. MPG-PROTO will help address this last question, while the work being carried out at Johnson Space Center on avionics development for MPG is addressing the second issue. Ultimately, to fully understand the degree to which $1-\mathrm{g}$ modal data is predictive of $0-\mathrm{g}$ fill levels, a full-scale, in-space test is required.

\section{ACKNOWLEDGEMENTS}

This work was supported under NASA grants \#80NSSC18K0272 and \#80NSSC18P1486 and through student funding provided by the NASA Wisconsin Space Grant Consortium. Support of the NASA Flight Opportunities Program, the ZERO-G Corporation, and the Carthage College Summer Undergraduate Research Experience program is gratefully acknowledged. The authors gratefully acknowledge significant contributions from Celestine Ananda, Bennett Bartel, Nicholas Bartel, Cassandra Bossong, Alec Digirolamo, Sheila Franklin, Megan Janiak, Nathan Lee, Taylor Peterson, and Jackson Wehr.

\section{REFERENCES}

Casanova S, de Frahan J, Goecks V, Vinicius Herath S, Martinez M, Jamieson N, Jones T, Kang S, Katz S, Li G, O'Sullivan D, Pastor D, Sharifrazi N, Sinkovec B, Sparta J, Vernacchia M (2017) Enabling deep space exploration with an in-space propellant depot supplied from lunar ice. In AIAA SPACE and Astronautics Forum and Exposition. http://dx.doi.org/10.2514/6.2017-5376.

Chu WH (1963) Breathing vibrations of a partially filled cylindrical tank - Linear theory, Journal of Applied Mechanics 30, 532-536.

Crosby K (2018) Modal propellant gauging: High-resolution and non-invasive gauging of both settled and unsettled liquids in reduced gravity. In Proceedings of the 69th Annual International Astronautical Congress, Bremen, Germany, 1-5 October 2018. IAC-18-C4.3.15x42358.

Crosby K, Werlink R, Hurlbert Er. (2020) Modal propellant gauging: Spectral density method. AIAA SciTech 2020 Forum. doi:10.2514/6.2020-1443, Available from: https://arc.aiaa.org/doi/ pdf/10.2514/6.2020-1443.

Crosby K, Williams N, Werlink R, Hurlbert E (2019) Modal propellant gauging: High-resolution and non-invasive gauging of both settled and unsettled liquids in reduced gravity. Acta Astronautica 159. http://dx.doi.org/10.1016/j.actaastro.2019.01.050.

Crosby KM, Rundle T, LeCaptain K, Werlink R (2016) Modal propellant gauging in low gravity. In AIAA SPACE 2016. AIAA SPACE Forum, (AIAA 2016-5533) http://dx.doi.org/10.2514/6.2016-5533. 
Dassault Systems (2019) Solidworks 2019. Available from: https:// www.solidworks.com/

Dodge F (2008) Propellant Mass Gauging: Database of Vehicle Applications and Research and Development Studies. NASA/CR2008215281.

Fahy F, Gardonio P (2007) Sound and Structural Vibration: Radiation, Transmission, and Response, 2nd edn, Elsevier. 978-0-12373633-8.

Hirt CW, Nichols BD (1981) Volume of fluid (VOF) method for the dynamics of free boundaries. Journal of Computational Physics 39(1), 201-225. Bibcode:1981JCoPh..39..201H. doi:10.1016/0021-9991(81)90145-5.

Holmes M (2019) Satellite Servicing Becomes and Actual Market. Satellite Today. March 2019. Available from: http://interactive. satellitetoday.com/via/march-2019/satellite-servicing-becomesanactual-market/

Ibrahim F (2005) Liquid Sloshing Dynamics Theory and Applications. United Kingdom: Cambridge University Press CB2 8BS.

MPG P9 Flight Video (2019) Available from: https://youtu.be/netd2QPiWk

National Aeronautics and Space Administration (2015) 2015 NASA Technology Roadmaps TA2: In Space Propulsion Technologies. Available from: https://www.nasa.gov/sites/default/files/atoms/ files/2015_nasa_technology_roadmaps_ta_2_in-space_ propulsion_final.pdf

National Aeronautics and Space Administration (2020) 2020 NASA Technology Taxonomy. Available from: https://www.nasa.gov/ offices/oct/taxonomy/index.html.

Notardonato W, Johnson W, Swanger A, McQuade W (2012) Inspace propellant production using water. In AIAA SPACE 2012. http://dx.doi.org/10.2514/6.2012-5288.

OpenFoam, Ltd. (2020) Available from: https://www.openfoam.com/.

Press WH, Teukolsky SA, Vetterling WT, Flannery BP (1992) Numerical Recipes in C, 2nd edn, Cambridge University Press.

Whitley R, Martinez R (2016) Options for staging orbits in cislunar space. In 2016 IEEE Aerospace Conference, Big Sky, MT, 2016, pp. 1-9, http://dx.doi.org/10.1109/AERO.2016.7500635.

Yendler B (2006) Review of propellant gauging methods. In 44th AIAA Aerospace Sciences Meeting and Exhibit, American Institute of Aeronautics and Astronautics, January 2006, pp. 1-7.

Zimmerli G, Asipauskas M, Wagner J, Follo J (2011). Propellant quantity gauging using the radio frequency mass gauge. In 49th AIAA Aerospace Sciences Meeting. http://dx.doi. org/10.2514/6.2011-1320. 